\section{Pocket-creation method of endoscopic submucosal dissection to achieve en bloc resection of giant colorectal subpedunculated neoplastic lesions}

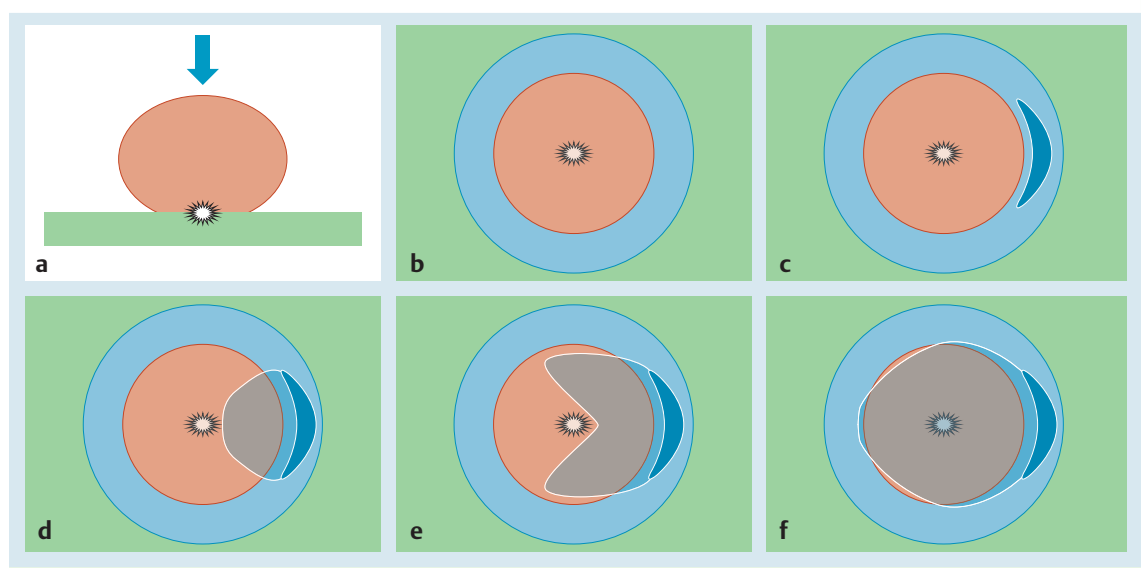

Fig. 1 Illustration showing the sequence of the pocket-creation method for endoscopic submucosal dissection of a giant, subpedunculated, neoplastic lesion. a A lesion with severe fibrosis at the center (lateral view). b A view of the same lesion from the top after submucosal injection. $\mathbf{c}$ Initial mucosal incision. $\mathbf{d}$ Creation of a submucosal pocket. e Extension of the submucosal pocket on both sides of the fibrosis. $\mathbf{f}$ Dissection of the fibrosis.
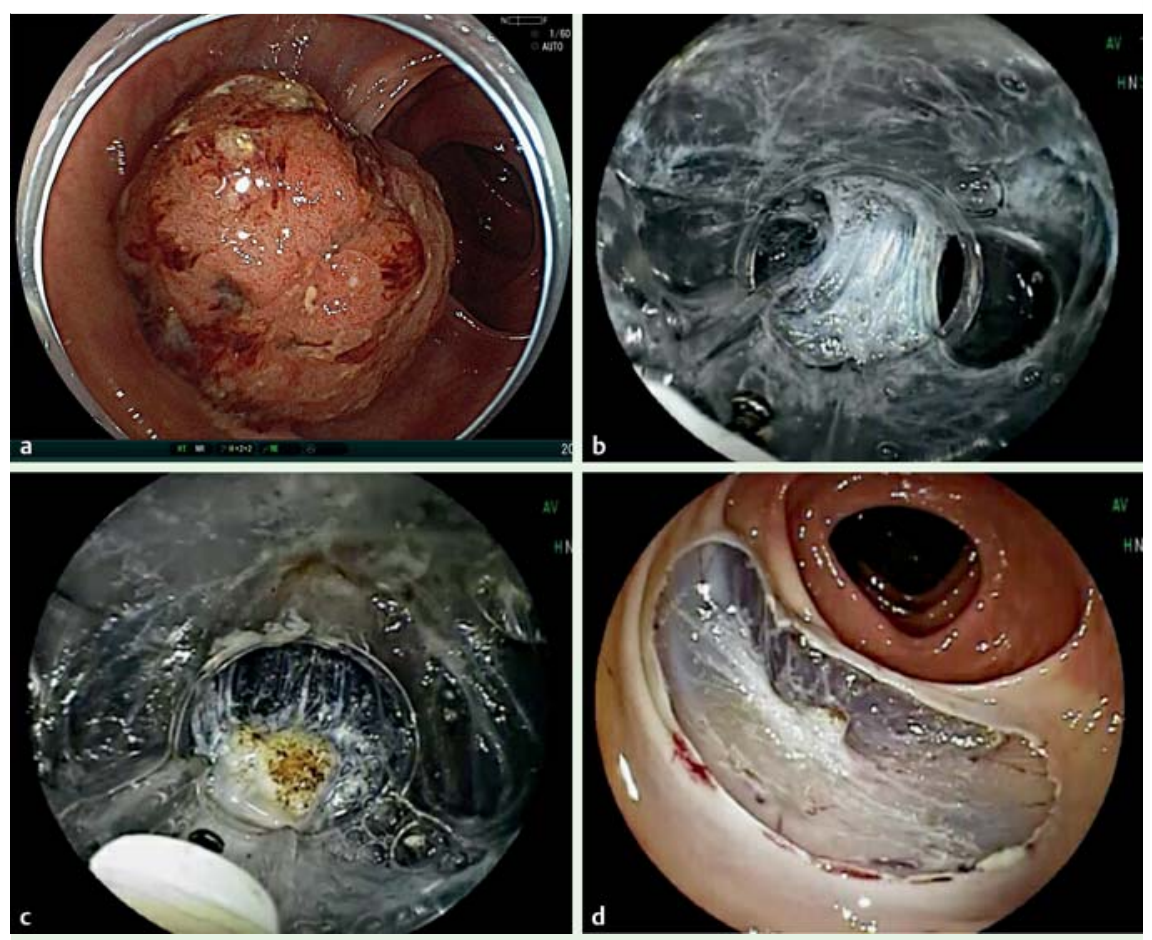

Fig. 2 Endoscopic images of the pocket-creation method of endoscopic submucosal dissection (ESD) for giant, subpedunculated, neoplastic lesions with severe fibrosis. a Endoscopic view of a subpedunculated, neoplastic lesion, $36 \mathrm{~mm}$ in diameter, in the ascending colon. b Severe fibrosis between the center of the lesion and the muscle layer after extension of the submucosal pocket on both sides. $\mathbf{c}$ The muscle layer was freed from the lesion after cutting the fibrotic area. $\mathbf{d}$ The muscle layer was flattened after ESD.
Endoscopic submucosal dissection (ESD) has become a standard minimally invasive therapy in Japan for large, superficial, colorectal neoplasms [1]. Although polypectomy or endoscopic mucosal resection is generally effective for polypoid lesions, ESD is an effective alternative to achieve en bloc resection in difficult cases, such as giant, subpedunculated, neoplastic lesions that are at least $3 \mathrm{~cm}$ in diameter [2]. However, ESD for giant, subpedunculated, neoplastic lesions is associated with technical difficulties because severe submucosal fibrosis occurs at the center of these lesions due to prolapse, resulting in the muscle layer being pulled upward. The pocket-creation method (PCM) is a new ESD strategy to overcome these difficulties ( Fig. 1; Video 1). The key feature of PCM is the creation of a large submucosal pocket under the lesion using a small-caliber tip transparent hood [3]. PCM has two advantages: maintenance of the thick submucosal layer with a minimal mucosal incision, which prevents the leakage of injection solution, and provision of good traction, which allows the submucosal tissue to be stretched and facilitates submucosal dissection.

PCM is very useful for ESD of giant, subpedunculated, neoplastic lesions with severe fibrosis ( Fig. 2). If there is severe fibrosis at the center of the lesion, submucosal pockets should be created on both sides of the fibrosis. By creating pockets on both sides of the fibrosis, the muscle layer can be clearly recognized. The muscle layer is pulled up at the fibrotic area, thus forming the shape of a mountain ( Fig.2b). By using the small-caliber tip transparent hood and creating the pockets, the submucosal space is opened near the fibrotic area. Dissection of the fibrosis can then be easily achieved and the lesion is freed from the muscle layer ( $\bullet$ Fig.2c). After this, only dissection of the remaining mucosa is needed ( Fig. 2 d; $\bullet$ Fig. 3 ).

PCM of ESD safely achieves an en bloc and complete resection of giant, subpedunculated, neoplastic lesions with severe fibrosis.

\section{Video 1}

The pocket-creation method of endoscopic submucosal dissection for a giant, subpedunculated, neoplastic lesion with severe fibrosis. 


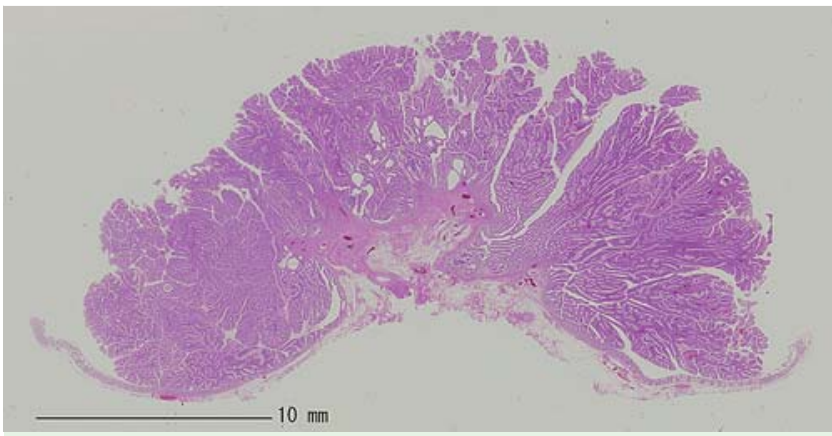

Fig. 3 Histology of the endoscopic submucosal dissection specimen showed a well-differentiated tubular adenocarcinoma in a high grade tubular adenoma (hematoxylin and eosin, Loupe image). The carcinoma was intramucosal cancer with no lymphovascular invasion and negative resection margins.
Competing interests: None

\section{Yoshikazu Hayashi ${ }^{1}$, Keijiro Sunada', Haruo Takahashi ${ }^{1}$, Hakuei Shinhata ${ }^{1}$, Alan T. Lefor ${ }^{2}$, Akira Tanaka ${ }^{3}$, Hironori Yamamoto ${ }^{1}$}

${ }^{1}$ Department of Medicine, Division of Gastroenterology, Jichi Medical University, Shimotsuke, Japan

${ }^{2}$ Department of Surgery, Jichi Medical University, Shimotsuke, Japan

${ }^{3}$ Department of Pathology, Jichi Medical University, Shimotsuke, Japan
Bibliography

DOI http://dx.doi.org/

10.1055/s-0034-1377438

Endoscopy 2014; 46: E421-E422

(c) Georg Thieme Verlag KG

Stuttgart · New York

ISSN 0013-726X

\section{Corresponding author} Hironori Yamamoto, MD, PhD

Department of Medicine Division of Gastroenterology Jichi Medical University 3311-1 Yakushiji Shimotsuke Tochigi, 329-0498 Japan

Fax: +81-285-448297

yamamoto@jichi.ac.jp 